\title{
Correlation between E-cadherin and p120 expression in invasive ductal breast cancer with a lobular component and MRI findings
}

\author{
Mary-Ann EI Sharouni ${ }^{1}$ Emily L. Postma ${ }^{2} \cdot$ Paul J. van Diest ${ }^{1,3}$
}

Received: 28 March 2017 /Revised: 23 June 2017 / Accepted: 12 July 2017 / Published online: 4 August 2017

(C) The Author(s) 2017. This article is an open access publication

\begin{abstract}
Invasive breast cancer comprises a spectrum of histological changes with purely lobular cancer on one side and purely ductal cancer on the other, with many mixed lesions in between. In a previous study, we showed that in patients with any percentage lobular component at core needle biopsy, preoperative MRI leads to the detection of clinically relevant additional findings in a substantial percentage of patients, irrespective of the percentage of the lobular component. Detection of a small lobular component may however not be reproducible among pathologists. Loss of membrane expression of E-cadherin or p120 is useful biomarkers of ILC and may therefore support a more objective diagnosis. All patients diagnosed with breast cancer containing a lobular component of any percentage between January 2008 and October 2012 were prospectively offered preoperative MRI. Clinically relevant additional findings on MRI were verified by pathology evaluation. Expression patterns of Ecadherin and p120 were evaluated by immunohistochemistry on the core needle biopsy. MRI was performed in 109 patients. The percentage of lobular component was significantly increased in cases with aberrant E-cadherin or p120 expression (both $p=<0.001$ ). However, aberrant expression of E-cadherin and p120 was not related to the probability of detecting relevant additional MRI findings. E-
\end{abstract}

Paul J. van Diest

p.j.vandiest@umcutrecht.nl

1 Department of Pathology, University Medical Centre Utrecht, PO Box 85500, 3508 GA Utrecht, The Netherlands

2 Department of Surgery, University Medical Centre Utrecht, PO Box 85500, 3508 GA Utrecht, The Netherlands

3 Department of Pathology, University Medical Centre Utrecht, Heidelberglaan 100, 3584 CX Utrecht, The Netherlands cadherin and p120 did not appear to be useful objective biomarkers for predicting additional relevant findings on MRI in patients with a lobular component in the core needle of their breast cancer.

Keywords Breast neoplasms · Magnetic resonance imaging · Biomarkers · Lobular

\section{Introduction}

Invasive breast cancer comprises a wide spectrum of specific histological types with varying clinical presentation, imaging characteristics, behavior and response to treatment. The histological spectrum of invasive breast cancers is a biological continuum with gradual transitions between the different types, e.g., invasive carcinomas with both ductal and lobular histological features frequently occur ("ductolobular," IDLC). Invasive lobular carcinoma (ILC) often has a diffuse infiltrative growth pattern without microcalcifications and has a tendency to present in both breasts. Because of this, the extent of the lesion is frequently underestimated by mammography or ultrasound, increasing the risk of positive margins at breast surgery [1-7]. MRI has shown to be of added value for estimation of the extent of the lesion in ILC [2, 8, 9]. Patients with a core biopsy diagnosis of ILC therefore routinely undergo preoperative magnetic resonance imaging (MRI).

Recently, we showed that preoperative MRI in patients with IDLC yields clinically relevant additional finding in a substantial number of patients, irrespective of the percentage of the lobular component [9]. Since detecting a small lobular component may not be reproducible between pathologists, we searched for more objective biomarkers to 
determine if a preoperative MRI may be indicated in this selected patient category.

E-cadherin is the main cell-to-cell-adhesion molecule in the adherens junction of epithelial cells and its expression is very often lost in ILC by somatic mutations, deletions or promoter methylation. Because of this E-cadherin expression is a useful marker in the distinction between ILC and infiltrating ductal cancer (IDC) [10-12]. However, since only one allele may be affected with only partial loss of membrane expression, interpretation may be challenging in some cases. In literature, p120 has been suggested as another diagnostic marker in the distinction between ILC and IDC [13]. p120 is a catenin and also an essential component of adherens junctions. It is thought to be a key regulator of E-cadherin function, stability, and strength by directly influencing the adhesive strength by controlling the amount of cadherin available at the cell surface for adhesion [14-16]. Catenins respond differently to functional loss of E-cadherin. In ILC, p120 accumulates in the cytoplasm, whereas in IDC it is located on the membrane [13, 17, 18]. p120 may therefore be regarded as a useful diagnostic biomarker for lobular differentiation, next to E-cadherin.

We therefore set out to determine the value of E-cadherin and p120 expression patterns as potential objective biomarkers for predicting additional relevant findings on preoperative MRI in patients with invasive breast cancer with a lobular component of any size on core needle biopsy.

\section{Materials and methods}

Between January 2008 and October 2012, all patients diagnosed with an invasive breast carcinoma showing lobular features on core needle biopsy were offered preoperative MRI as previously described [9]. Radiology reports of conventional imaging and MRI were compared and additional findings detected on MRI were registered as previously described [9].

All core needle biopsies were neutral buffered formaldehyde fixed, processed and paraffin embedded according to standard protocols. A dedicated breast cancer pathologist (PJvD) reviewed all slides and the percentage of lobular component was determined as previously described [9]. Surgical breast specimens were routinely processed.

E-cadherin and p120 were stained by immunohistochemistry on an automated immunostainer (BondMax, Leica) according to the manufacturer's instructions. An experienced observer (PJvD) scored E-cadherin membrane staining as normal, reduced or negative, and p120 was scored as normal (membrane staining comparable to normal breast tissue), reduced or aberrant (cytoplasmic/negative) staining. Figure 1 shows examples of normal and aberrant staining for Ecadherin and p120.

\section{Statistical analysis}

Data were analyzed using SPSS version 17.0. Normally distributed continuous variables were presented as means (standard deviation) and compared with independent $t$ test. Notnormally distributed data were presented as medians (range) and compared with the Mann-Whitney U test. The chi-square test was used to compare percentages. A $p$-value $<0.05$ was considered significant. Patients were arbitrarily stratified into groups with different percentages of the lobular component; 0-30\%, 31-70\%, and 71-100\% as before [9]. The CochranArmitage test was used to test for trend. Reduced E-cadherin and p120 staining was grouped with normal staining.

\section{Results}

\section{Patients and imaging}

Of the 505 patients diagnosed with invasive breast carcinoma, lobular differentiation was reported in 155 (31\%) patients, ranging from 1 to $100 \%$ of lobular component at core needle biopsy. Patients whose MR-images were available $(n=109)$ were included in this analysis. The remaining 46 patients had either not undergone for MRI because of claustrophobia and obesity or had inadequate MR-imaging. All included patients were women with a mean age of 57.5 years. Seventy-two percent of the tumors were palpable. For a full table with baseline characteristics, we refer to our previous paper [9].

\section{Additional foci on MRI}

As previously described, the quality of the slide was too low in one patient to be assessed for percentage of lobular component. Preoperative MRI detected additional malignant ("relevant MRI") finding in 47/108 (44\%) patients, 28/47 (60\%) with additional ipsi- or contralateral foci, and more extensive disease $(\geq 5.0 \mathrm{~mm})$ in $19 / 47$ (40\%) patients [9]. Paraffin blocks in 99/109 (91\%) had sufficient residual invasive cancer to perform E-cadherin and p120 staining.

\section{E-cadherin and p120 and lobular component}

E-cadherin was aberrant in 39/109 (39\%) and p120 was aberrant in 29/109 (35\%) of cases. The percentage lobular component was significantly higher in E-cadherin aberrant cases than in E-cadherin normal/reduced cases ( $p=<0.001$, Fig. 2). Likewise, the percentage lobular component was significantly higher in p120 aberrant cases than in p120 normal/reduced cases ( $p=<0.001$, Fig. 3 ). As shown in Table 1, neither Ecadherin nor p120 status could predict additional relevant findings in the preoperative MRI in the study population, also not when stratified for the percentage of the lobular 
Fig. 1 Examples of normal and aberrant staining for E-cadherin and p120 all at $10 \times$ magnification

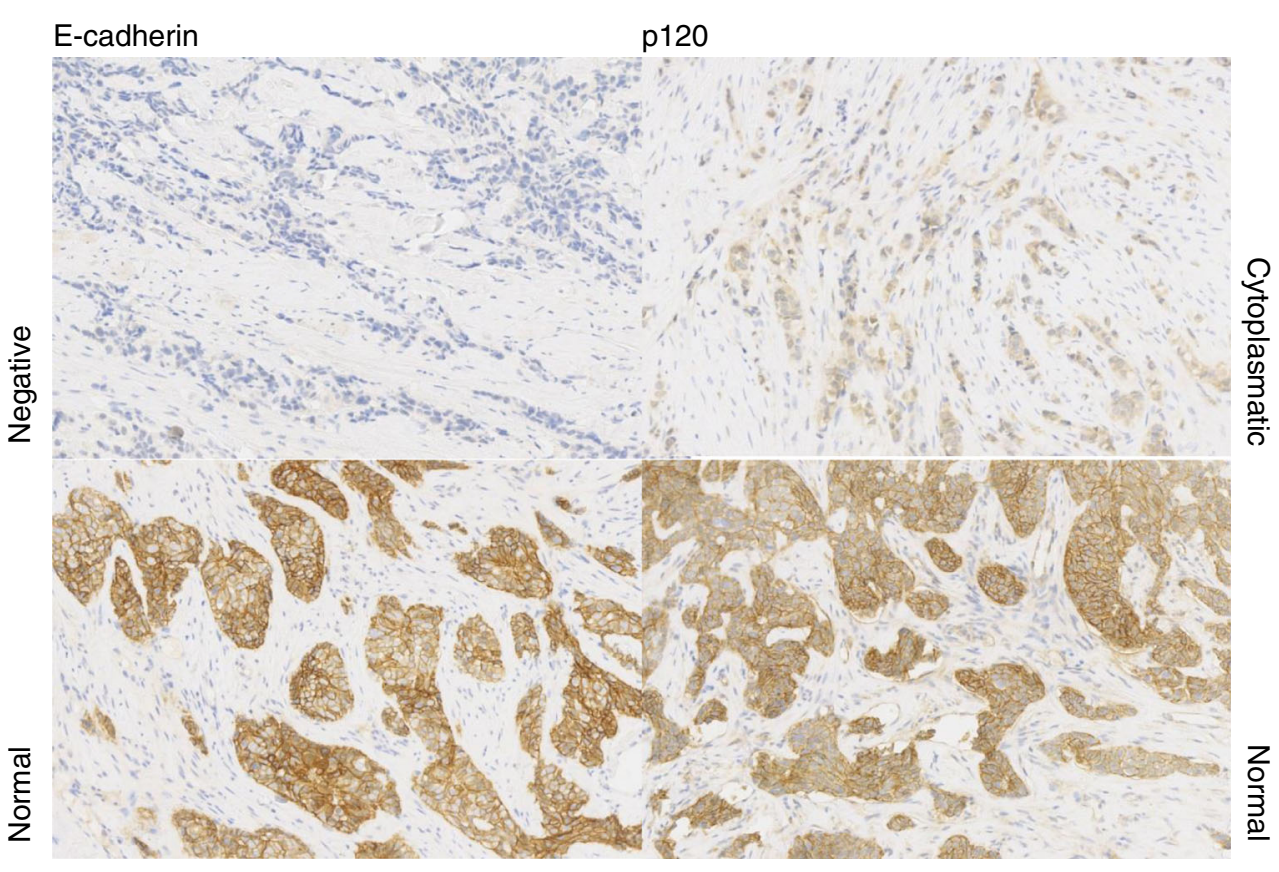

component ( 100 vs. $<100 \%$ in Table $21-30 \%$ vs. $31-70 \%$ vs. $71-100 \%$ in Table 3). Similarly, when subdividing the group of additional relevant findings into the group with patients with additional malignant foci and the group with more extensive disease, neither E-cadherin nor p120 status could predict additional relevant findings in the preoperative MRI (data not shown). Grouping the cases with reduced staining ( 7 for $\mathrm{p} 120$, 1 for E-cadherin) with normal or aberrant staining did not influence the results (data not shown).

\section{Discussion}

In this study, we showed that E-cadherin and p120 do not appear to be useful objective biomarkers for defining presence of lobular component in a core needle biopsy in patients with IDLC. Nor does it predict for additional relevant findings on MRI. Patients with ILC routinely undergo MRI in order to better visualize the extent of the index lesion and identify contralateral lesions which occur at
Fig. 2 Lobular component in relation to E-cadherin expression pattern in 109 patients with ductolobular breast cancer $(p=<0.001)$

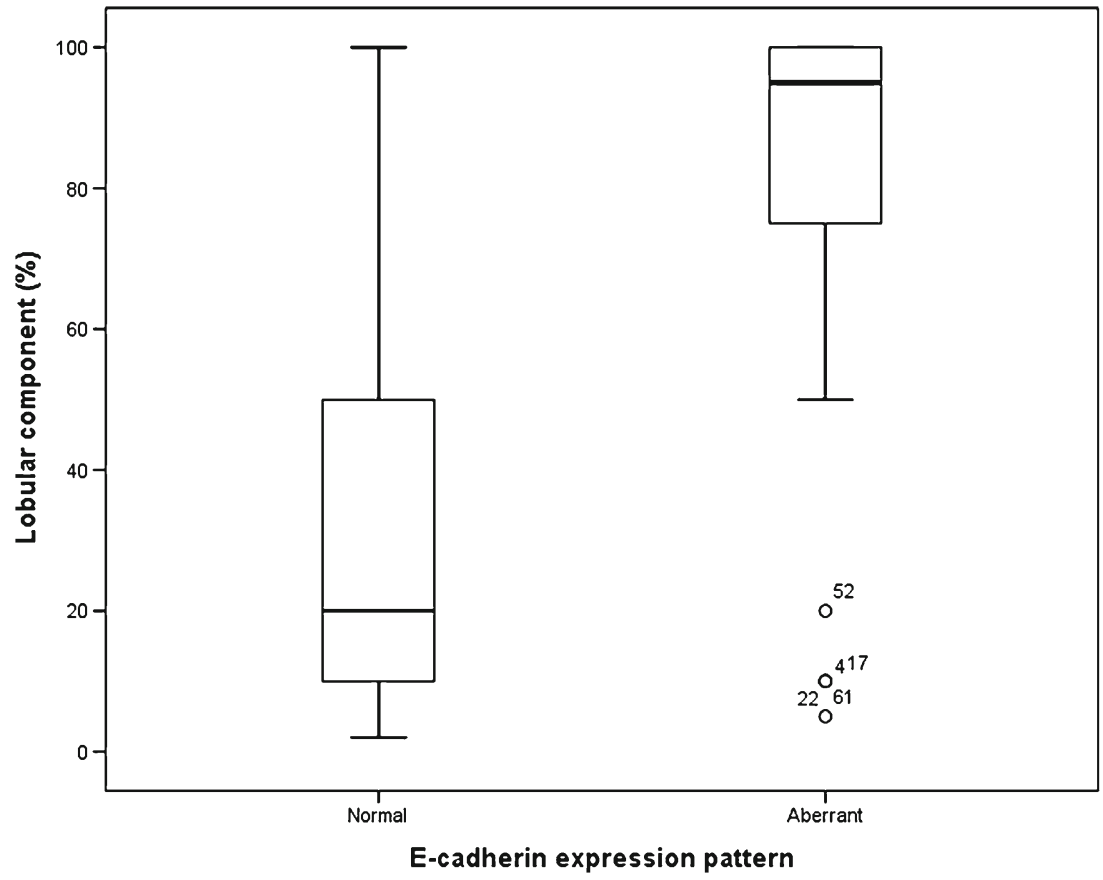


Fig. 3 Lobular component in relation to $\mathrm{p} 120$ expression pattern in 109 patients with ductolobular breast cancer $(p=<0.001)$

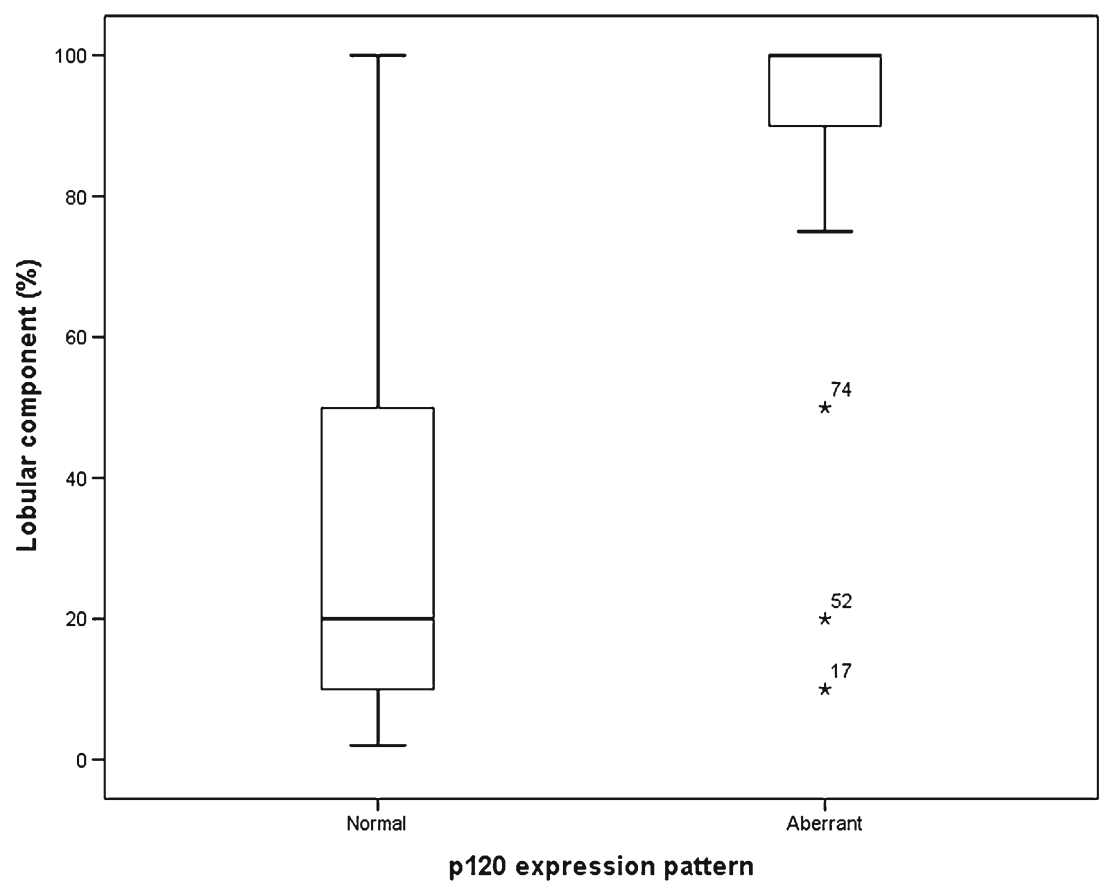

increased frequency. Ductolobular breast cancer has features of both ductal and lobular breast cancer, and biologically, one could expect an increased frequency of bilateral and/or ipsilateral additional foci in case of a smaller lobular component, as is the case for pure ILC. Indeed, we recently we showed for the first time that additional malignant MRI findings are found at the same frequency in ductal breast carcinoma with the smallest lobular component, starting from $2 \%$, as in ductal breast carcinoma with a major lobular component [9].

The WHO defines mixed breast cancer subtypes as types that have "a specialized pattern in at least $50 \%$ of the tumor and a non-specialized pattern in between $10 \%$ and 49\%." [19]. Few studies consider IDLC a distinct type of breast cancer, but according to several studies, it is a carcinoma with mixed differentiation best to be compared

Table 1 Probability of detecting clinically relevant additional MRI findings in breast cancer patients with any percentage lobular differentiation on core needle biopsy stratified for E-cadherin and p120 status

\begin{tabular}{llllll}
\hline Additional MRI findings & \multicolumn{2}{l}{ E-cadherin expression } & & p120 expression \\
\cline { 2 - 3 } \cline { 5 - 6 } & Normal & Aberrant & & Normal & Aberrant \\
\hline $\begin{array}{l}\text { Relevant } \\
\begin{array}{l}\text { None or } \\
\quad \text { not relevant }\end{array}\end{array}$ & $24(40 \%)$ & $19(49 \%)$ & & $29(41 \%)$ & $14(48 \%)$ \\
$\begin{array}{l}\text { Total } \\
n\end{array}$ & $36(60 \%)$ & $20(51 \%)$ & & $41(59 \%)$ & $15(52 \%)$ \\
& $60(61 \%)$ & $39(39 \%)$ & $70(65 \%)$ & $29(35 \%)$ \\
& $p=0.258$ & & $p=0.342$ & \\
\hline
\end{tabular}

with ILC $[6,7,20]$. A recent study by Arps et al. is perhaps the first article published considering ductal carcinoma with lobular component as a distinct type of breast cancer. They retrospectively selected 183 patients with IDLC breast cancer and quantified the lobular component into categories per $20 \%$. The compared clinical and pathological data of these patients were compared to 1499 patients with ductal breast cancer and 375 patients with ILC. Their data confirm previous research and suggest that the clinical and biological characteristics of IDLC are more similar to that of ILC [21].

Table 2 Probability of detecting clinically relevant additional MRI findings in breast cancer patients with varying percentage of lobular differentiation on core needle biopsy, stratified for E-cadherin and p120 status

\begin{tabular}{|c|c|c|c|c|}
\hline \multirow[t]{2}{*}{$\%$ lobular component } & \multicolumn{2}{|c|}{ E-cadherin expression } & \multicolumn{2}{|c|}{ p120 expression } \\
\hline & Normal & Aberrant & Normal & Aberrant \\
\hline \multicolumn{5}{|l|}{$1-99 \%$} \\
\hline Relevant & $23(41 \%)$ & $11(50 \%)$ & $26(41 \%)$ & $8(57 \%)$ \\
\hline \multirow{2}{*}{$\begin{array}{l}\text { None or } \\
\text { not relevant }\end{array}$} & $33(59 \%)$ & $11(50 \%)$ & $38(59 \%)$ & $6(43 \%)$ \\
\hline & $p=0.321$ & & $p=0.202$ & \\
\hline \multicolumn{5}{|l|}{$100 \%$} \\
\hline Relevant & $1(25 \%)$ & $7(44 \%)$ & $2(40 \%)$ & $6(40 \%)$ \\
\hline \multirow{2}{*}{$\begin{array}{l}\text { None or } \\
\text { not relevant }\end{array}$} & $3(75 \%)$ & $9(56 \%)$ & $3(60 \%)$ & $9(60 \%)$ \\
\hline & $p=0.465$ & & $p=0.704$ & \\
\hline
\end{tabular}


Table 3 Probability of detecting clinically relevant additional MRI findings in breast cancer patients with varying percentages lobular differentiation on core needle biopsy, stratified for E-cadherin and p120 status

\begin{tabular}{|c|c|c|c|c|}
\hline \multirow[t]{2}{*}{$\%$ lobular component } & \multicolumn{2}{|c|}{ E-cadherin expression } & \multicolumn{2}{|c|}{ p120 expression } \\
\hline & Normal & Aberrant & Normal & Aberrant \\
\hline \multicolumn{5}{|l|}{$1-30 \%$} \\
\hline Relevant & $14(38 \%)$ & $3(60 \%)$ & $15(38 \%)$ & $2(100 \%)$ \\
\hline \multirow[t]{2}{*}{ None or not relevant } & $23(62 \%)$ & $2(40 \%)$ & $25(62 \%)$ & $0(0 \%)$ \\
\hline & $p=0.317$ & & $p=0.158$ & \\
\hline \multicolumn{5}{|l|}{$31-70 \%$} \\
\hline Relevant & $5(50 \%)$ & $0(0 \%)$ & $5(42 \%)$ & $0(0 \%)$ \\
\hline \multirow[t]{2}{*}{ None or not relevant } & $5(50 \%)$ & $3(100 \%)$ & $7(58 \%)$ & $1(100 \%)$ \\
\hline & $p=0.196$ & & $p=0.615$ & \\
\hline \multicolumn{5}{|l|}{$71-100 \%$} \\
\hline Relevant & $5(38 \%)$ & $15(50 \%)$ & $8(47 \%)$ & $12(46 \%)$ \\
\hline \multirow[t]{3}{*}{ None or not relevant } & $8(62 \%)$ & $15(50 \%)$ & $9(53 \%)$ & $14(54 \%)$ \\
\hline & $p=0.360$ & & $p=0.600$ & \\
\hline & $C A=p=0.942$ & & $C A=0.266$ & \\
\hline
\end{tabular}

CA Cochrane armitage trend test.
Diagnosing IDLC is however subjective and observer dependent, and thereby probably not optimally reproducible. In our study, in 24/109 patients, the lobular component was at revision classified as $100 \%$ at revision, suggesting these patients might not have been IDLC but ILC instead. This is within normal limits of inter-observer variability with regard to histologic typing in pathology, and conveniently allowed evaluation of MRI findings over the full spectrum from IDLC towards ILC. In order to find an objective biomarker for this selected group of patients, we investigated the expression of E-cadherin and p120, two proteins involved in adherens junction function which is often impaired in ILC [18, 22]. However, it should be noted that staining of E-cadherin and p120 is also dependent on technical details of the immunostaining protocol, which is why we used the normal surrounding breast parenchyma as internal control. Our results show that neither E-cadherin nor p120 is associated with additional malignant MRI findings in patients with IDLC, similar when stratifying for the percentage of the lobular component. Nevertheless, the percentage of the lobular component was significantly higher for cases with aberrant E-cadherin or p120 expression, consistent with previous research where both are biomarkers of lobular differentiation [13, 17, 18, 23]. About 40 and $30 \%$ of our IDLC cases had abnormal Ecadherin and p120 expression, respectively, confirming the in between nature of these cancers in the spectrum from pure IDC to pure ILC.

We agree that $80 \%$ of ILC showing aberrant E-cadherin expression is on the low side, but the usually higher numbers are based on resections rather than on biopsies as in the present study.

In conclusion, E-cadherin and p120 expression do not appear to be useful objective biomarkers for predicting additional relevant finding on MRI in patients with mixed ductal/lobular differentiation in core needle biopsies of their breast cancer.

Compliance with ethical standards Anonymous use of redundant tissue for research purposes is part of the standard treatment agreement with patients in our hospital [24].

Funding None, not applicable.

Conflict of interest The authors declare that they have no conflict of interest.

Open Access This article is distributed under the terms of the Creative Commons Attribution 4.0 International License (http:// creativecommons.org/licenses/by/4.0/), which permits unrestricted use, distribution, and reproduction in any medium, provided you give appropriate credit to the original author(s) and the source, provide a link to the Creative Commons license, and indicate if changes were made.

\section{References}

1. Boetes C, Veltman J, van Die L, Bult P, Wobbes T, Barentsz JO (2004) The role of MRI in invasive lobular carcinoma. Breast Cancer Res Treat 86(1):31-37. doi:10.1023/B:BREA. 0000032921.10481.dc

2. Mann RM, Hoogeveen YL, Blickman JG, Boetes C (2008) MRI compared to conventional diagnostic work-up in the detection and evaluation of invasive lobular carcinoma of the breast: a review of existing literature. Breast Cancer Res Treat 107(1):1-14. doi:10. 1007/s10549-007-9528-5

3. Kim SH, Cha ES, Park CS et al (2011) Imaging features of invasive lobular carcinoma: comparison with invasive ductal carcinoma. Jpn J Radiol 29(7):475-482. doi:10.1007/s11604-011-0584-8

4. Molland JG, Donnellan M, Janu NC, Carmalt HL, Kennedy CW, Gillett DJ (2004) Infiltrating lobular carcinoma - a comparison of 
diagnosis, management and outcome with infiltrating duct carcinoma. Breast 13(5):389-396. doi:10.1016/j.breast.2004.03.004

5. Quan ML, Sclafani L, Heerdt AS, Fey JV, Morris EA, Borgen PI (2003) Magnetic resonance imaging detects unsuspected disease in patients with invasive lobular cancer. Ann Surg Oncol 10(9):1048-1053

6. Rakha EA, Gill MS, El-Sayed ME et al (2009) The biological and clinical characteristics of breast carcinoma with mixed ductal and lobular morphology. Breast Cancer Res Treat 114(2):243-250. doi: 10.1007/s10549-008-0007-4

7. Suryadevara A, Paruchuri LP, Banisaeed N, Dunnington G, Rao KA (2010) The clinical behavior of mixed ductal/lobular carcinoma of the breast: a clinicopathologic analysis. World J Surg Oncol 8:51. doi:10.1186/1477-7819-8-51

8. Mann RM (2010) The effectiveness of MR imaging in the assessment of invasive lobular carcinoma of the breast. Magn Reson Imaging Clin N Am 18(2):259-276, ix. doi:10.1016/j.mric.2010. 02.005

9. El Sharouni MA, Postma EL, Menezes GL et al (2015) High prevalence of MRI-detected contralateral and ipsilateral malignant findings in patients with invasive ductolobular breast cancer: impact on surgical management. Clin Breast Cancer 16(4):269-275. doi:10. 1016/j.clbc.2015.10.002

10. Derksen PW, Braumuller TM, van der Burg E et al (2011) Mammary-specific inactivation of E-cadherin and p53 impairs functional gland development and leads to pleomorphic invasive lobular carcinoma in mice. Dis Model Mech 4(3):347-358. doi:10. 1242/dmm.006395

11. De Leeuw WJ, Berx G, Vos CB et al (1997) Simultaneous loss of Ecadherin and catenins in invasive lobular breast cancer and lobular carcinoma in situ. J Pathol 183(4):404-411

12. Singhai R, Patil VW, Jaiswal SR, Patil SD, Tayade MB, Patil AV (2011) E-cadherin as a diagnostic biomarker in breast cancer. N Am J Med Sci 3(5):227-233. doi:10.4297/najms.2011.3227

13. Dabbs DJ, Bhargava R, Chivukula M (2007) Lobular versus ductal breast neoplasms: the diagnostic utility of p120 catenin. Am J Surg Pathol 31(3):427-437. doi:10.1097/01.pas.0000213386.63160.3f

14. Ireton RC, Davis MA, van Hengel J et al (2002) A novel role for p120 catenin in E-cadherin function. J Cell Biol 159(3):465-476. doi: $10.1083 /$ jcb. 200205115
15. Davis MA, Reynolds AB (2006) Blocked acinar development, Ecadherin reduction, and intraepithelial neoplasia upon ablation of p120-catenin in the mouse salivary gland. Dev Cell 10(1):21-31. doi:10.1016/j.devcel.2005.12.004

16. Reynolds AB, Roczniak-Ferguson A (2004) Emerging roles for p120-catenin in cell adhesion and cancer. Oncogene 23(48):79477956. doi:10.1038/sj.onc. 1208161

17. Sarrio D, Perez-Mies B, Hardisson D et al (2004) Cytoplasmic localization of p120ctn and E-cadherin loss characterize lobular breast carcinoma from preinvasive to metastatic lesions. Oncogene 23(19):3272-3283. doi:10.1038/sj.onc.1207439

18. Schackmann RC, van Amersfoort M, Haarhuis JH et al (2011) Cytosolic p120-catenin regulates growth of metastatic lobular carcinoma through Rock1-mediated anoikis resistance. J Clin Invest 121(8):3176-3188. doi:10.1172/JCI41695

19. Lakhani SR, Ellis IO et al (2012) World health organization classification of tumors of the breast. Lyon, France

20. Bharat A, Gao F, Margenthaler JA (2009) Tumor characteristics and patient outcomes are similar between invasive lobular and mixed invasive ductal/lobular breast cancers but differ from pure invasive ductal breast cancers. Am J Surg 198(4):516-519. doi:10.1016/j. amjsurg.2009.06.005

21. Arps DP, Healy P, Zhao L, Kleer CG, Pang JC (2013) Invasive ductal carcinoma with lobular features: a comparison study to invasive ductal and invasive lobular carcinomas of the breast. Breast Cancer Res Treat 138(3):719-726. doi:10.1007/s10549-013-2493-2

22. Schackmann RC, Klarenbeek S, Vlug EJ et al (2013) Loss of p120catenin induces metastatic progression of breast cancer by inducing anoikis resistance and augmenting growth factor receptor signaling. Cancer Res 73(15):4937-4949. doi:10.1158/0008-5472.CAN-130180

23. Derksen PW, Liu X, Saridin F et al (2006) Somatic inactivation of E-cadherin and p53 in mice leads to metastatic lobular mammary carcinoma through induction of anoikis resistance and angiogenesis. Cancer Cell 10(5):437-449. doi:10.1016/j.ccr.2006.09.013

24. van Diest PJ (2002) No consent should be needed for using leftover body material for scientific purposes. BMJ 325:648-651. doi:10. 1136/bmj.325.7365.648 